\title{
Old main-sequence turnoff photometry in the SMC: Star Formation History and Chemical Enrichment Law
}

\author{
Noelia E. D. Noël ${ }^{1}$, Carme Gallart ${ }^{1}$, Antonio Aparicio ${ }^{1}$, Sebastián L. \\ Hidalgo $^{2}$, Ricardo Carrera ${ }^{1}$, Edgardo Costa $^{3}$ and René A. Méndez ${ }^{3}$ \\ ${ }^{1}$ Instituto de Astrofísica de Canarias. 38200 La Laguna. Tenerife, Canary Islands. Spain \\ email: noelia@iac.es \\ ${ }^{2}$ University of Minnesota, Department of Astronomy, 116 Church St. S.E., Minneapolis, \\ MN 55455 \\ ${ }^{3}$ Departamento de Astronomía, Universidad de Chile, Casilla 36-D, Santiago, Chile
}

\begin{abstract}
We present deep ground-based $B$ and $R$ observations of 12 fields in the Small Magellanic Cloud (SMC). The resulting color-magnitude diagrams (CMDs) reach the oldest mainsequence (MS) turnoff at $\mathrm{M}_{R} \sim 3.5$ and reveal the stellar population differences between the part of the galaxy facing the Large Magellanic Cloud (LMC) and an area on the opposite side. In the Southern part of the galaxy, we found that there are still intermediate-age stars as far as 4 kpc from the SMC center. The Chemical Enrichment History (CEH) in one of our SMC fields is also presented.
\end{abstract}

Keywords. local group galaxies: individual (SMC) — galaxies: star formation history

\section{Introduction}

In spite of the increasing interest in studying the SMC, reflected by recent works (e.g. Dolphin et al. 2001; Harris \& Zaritsky 2004), there are still many gaps in our knowledge of this galaxy. To shed light on some of these, we present a progress report of a project aimed at studying the star formation history ( $\mathrm{SFH}$ ) in different parts of the SMC. We observed 12 fields during a four years campaign (2001-2004) using the 100-inch telescope at Las Campanas Observatory, Chile. The fields are located from $\sim 1 \mathrm{kpc}$ to $\sim 4 \mathrm{kpc}$ from the center of the SMC. We will show a preliminary analysis of the age and metallicity ranges in each of the fields through isochrone's comparison, while a quantitative analysis is underway through comparison with synthetic CMDs. We also present the CEH of a SMC field, located at $\sim 1 \mathrm{kpc}$ from the center, as obtained from our synthetic CMD analysis. This $\mathrm{CEH}$ is compared with the $\mathrm{CEH}$ found by Carrera (PhD thesis) for the same field using the CaII triplet, i.e., a completely independent method.

\section{The SMC Stellar Content}

The interpretation of CMDs of composite stellar population strongly relies on the stellar evolution models adopted (see Gallart, Zoccali, \& Aparicio 2005). For our purpose we used the BaSTI stellar evolutionary models (Pietrinferni et al. 2004). From the isochrones' overlap it is possible to constrain the range of metallicities in the SMC. However, the method we use to calculate the SFH, involving comparison with synthetic CMDs (see Noël et al. 2007, this conference), allows us to obtain a reliable, more detailed CEH as seen in the next section. 


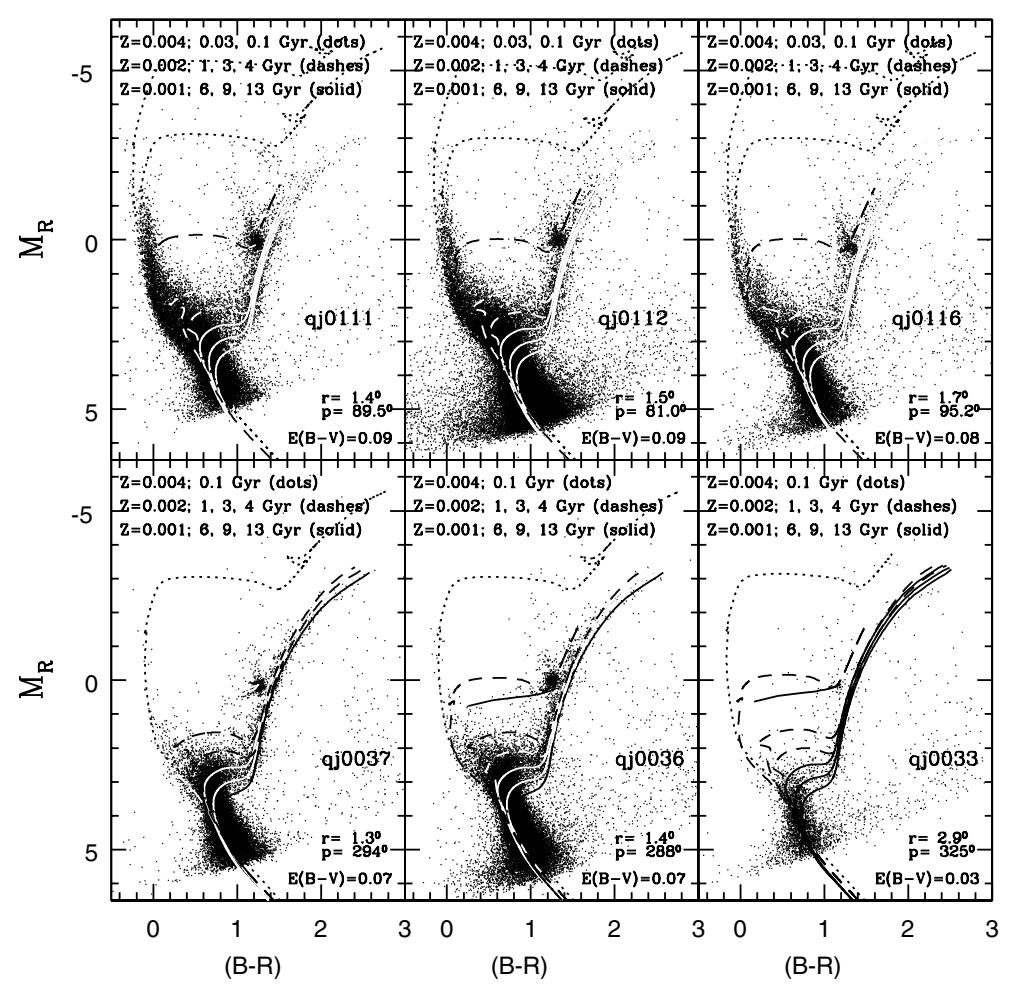

Figure 1. Upper panel: CMDs of the Eastern SMC fields. Lower panel: CMDs of the Western fields. See text for details.

Figures 1 and 2 show our SMC CMDs with isochrones overlapped. The CMDs of the different fields are displayed in order of increasing galactocentric distance. Position angles (p), reddening $\mathrm{E}(\mathrm{B}-\mathrm{V})$, metallicities and ages of the isochrones are labeled in both figures. A distance modulus $(\mathrm{m}-\mathrm{M})_{0}=18.9$ has been assumed.

The upper panel in Figure 1 shows the Eastern SMC CMDs. Note the conspicuous MS, which is well populated from the oldest turnoff at $\mathrm{M}_{R}=3.5$ up to the $0.03 \mathrm{Gyr}$ isochrone. All the CMDs show a large fraction of young stars $(<1$ Gyr old). The densely populated areas around the isochrones of age $>1$ Gyr and the extension in luminosity of the red clump indicate a strong presence of intermediate-age stars.

The lower panel in Figure 1 shows the Western SMC CMDs. It is noticeable that the star formation significatively dropped $\sim 3$ Gyr ago (see Noël et al. 2007). Even in the fields at larger galactocentric distances, the population is not purely old but dominated by intermediate-age stars, as indicated by the relatively high density of stars around the $3,4,6$, and 9 Gyr isochrones.

Figure 2 shows the CMDs corresponding to the Southern fields. Field smc0057 is the closest to the SMC center. Its CMD shows an important MS, with the area around the 0.1 Gyr isochrone still quite populated. The more distant fields toward the South are dominated by intermediate-age stars. These CMDs show a breaking point further than $\sim 2.7^{0}$, from where there are almost no stars younger than 3 Gyr old.

In all fields, the subgiant branch region is well populated from the oldest turnoffs up to the $\sim 1$ Gyr old turnoff, indicating that star formation has proceeded in a continuous 


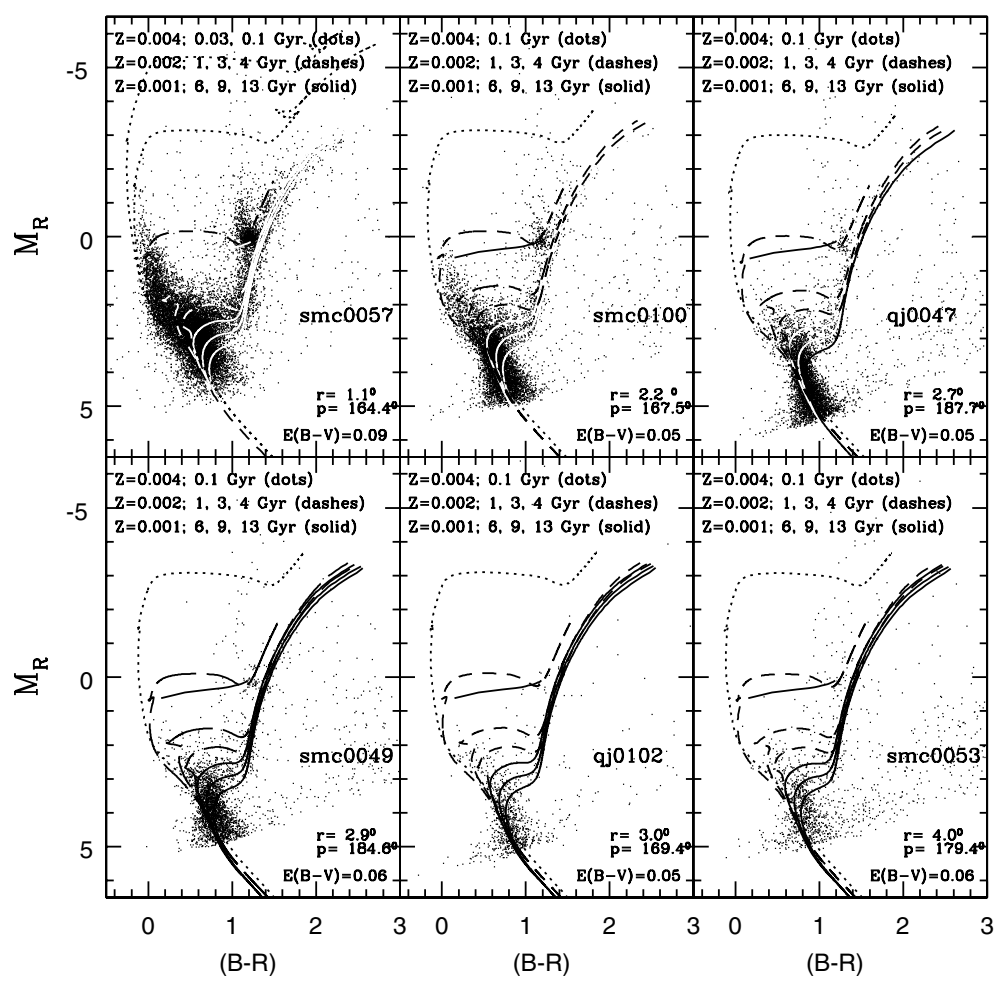

Figure 2. CMDs of the six Southern SMC fields.

way, with possible variations in intensity but no big gaps between successive bursts, over the galaxy's lifetime. The structure of the red clump of core He-burning stars is consistent with the large amount of intermediate-age population inferred from the MS and the subgiant branch region. The absence of a horizontal branch is a shared feature of all of the CMDs (see Noël et al. 2007 for details).

Our analysis shows that the underlying spheroidally distributed population is mainly composed by intermediate-age and old stars, and that its age distribution does not show strong galactocentric gradients.

\section{The Metallicity Law}

As explained in detail in Noël et al. (2007, this conference), we used the algorithm IAC-pop to solve the SFH of a SMC field (smc0057). The details of IAC-pop and its internal consistency are explained in Aparicio \& Hidalgo (2007, submitted). See Noël et al. (this conference) for a description of the process involved in the determination of the SFH of this particular field. The solution for the SFH provided by IAC-pop gives an independent determination of the $\mathrm{CEH}$ : each age range has an associated metallicity distribution. We have calculated the mean metallicity of the stars formed at each age interval. We plotted this mean metallicity obtained from the CMD modelling in Figure 3 (large stars), together with measurements of the metallicity of individual stars in the same field (small black squares), using CaII triplet spectroscopy, from Carrera (PhD Thesis). Our CEH agrees very well with the one obtained by Carrera using a completely 


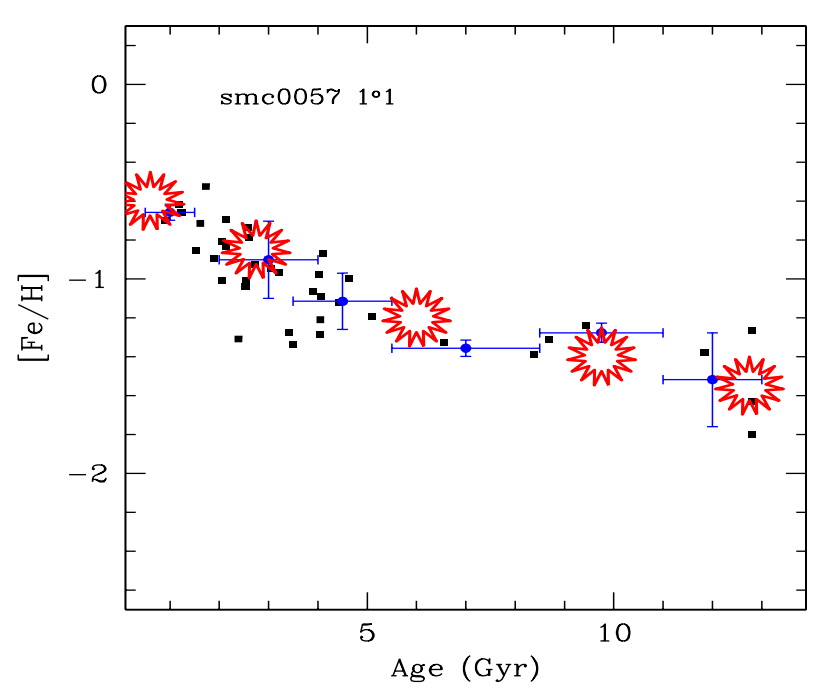

Figure 3. CEH of field smc0057. Small (black) squares: measurements of the metallicity of individual stars by Carrera (PhD Thesis), obtained from CaII triplet spectroscopy. Horizontal and vertical bars represent the metallicity dispersion corresponding to a given age range. Large stars (red symbols) represent our CEH obtained from the CMD modelling. Note the very good agreement of the two independent $\mathrm{CEH}$.

independent method. Thus, we have tested, for the first time, the external consistency of the IAC-pop algorithm. It should be stressed that we did not impose any a priori constraints on the CEH.

We found that the mean metallicity of stars formed in this SMC field remained relatively low $([\mathrm{Fe} / \mathrm{H}] \sim-1.3)$ until $\sim 4$ Gyr ago, at which point it began a steady increase to the present gas-phase abundance value of $[\mathrm{Fe} / \mathrm{H}] \sim-0.5$. The $\mathrm{CEH}$ found here is similar to the one found for clusters in the SMC (e.g. Pagel \& Tautvaišiené 1999), with the special feature that there is only one cluster older than 10 Gyr (NGC 121, Suntzeff et al. 1986), whereas in the field population there seems to be a significant fraction of old stars.

\section{References}

Dolphin, A., Walker, A. R., Hodge, P. W., Mateo, M., Olszewski, E. W., Schommer, R. A., \& Suntzeff, N. B. 2001, ApJ 562, 303

Gallart, C., Zoccali, M., \& Aparicio, A. 2005, ARA\&A 43, 387

Harris, J., \& Zaritsky, D. 2004, AJ 127, 1531

Noël, N. E. D., Gallart, G., Costa, E., \& Méndez, R. A. 2007, AJ in press

Pagel, B. E. J., \& Tautvaišiené, G. 1999, Ap\&SSS 265, 461

Pietrinferni, A., Cassisi, S., Salaris, M., \& Castelli, F. 2004, ApJ 612, 168

Suntzeff, N. B., Friel, E., Klemola, A., Kraft, R. P., \& Graham, J. A. 1986, AJ 91, 275

\section{Discussion}

GEISLER: How does your AMR compare to that of the star clusters from CaII triplet Kayser et al.)?.

Nö̈L: Our AMR is in good agreement with the one obtained using CaII triplet (for 50 stars) by Carrera (2006, Ph.D thesis) in the same SMC field. Our AMR was obtained 
without previous assumptions that is why it is so important this result. The AMR from Kayser et al. is in good agreement with the AMR by Carrera et al. and so with ours.

VAN DER MAREL: You mentioned that your data will be used also to estimate the proper motion of the SMC. Do you have a result from that?.

Nö̈L: My collaborators R. Méndez and E. Costa are working on the absolute proper motions in the Magellanic Clouds and as far as I know they have results from the LMC and preliminary results on the SMC.

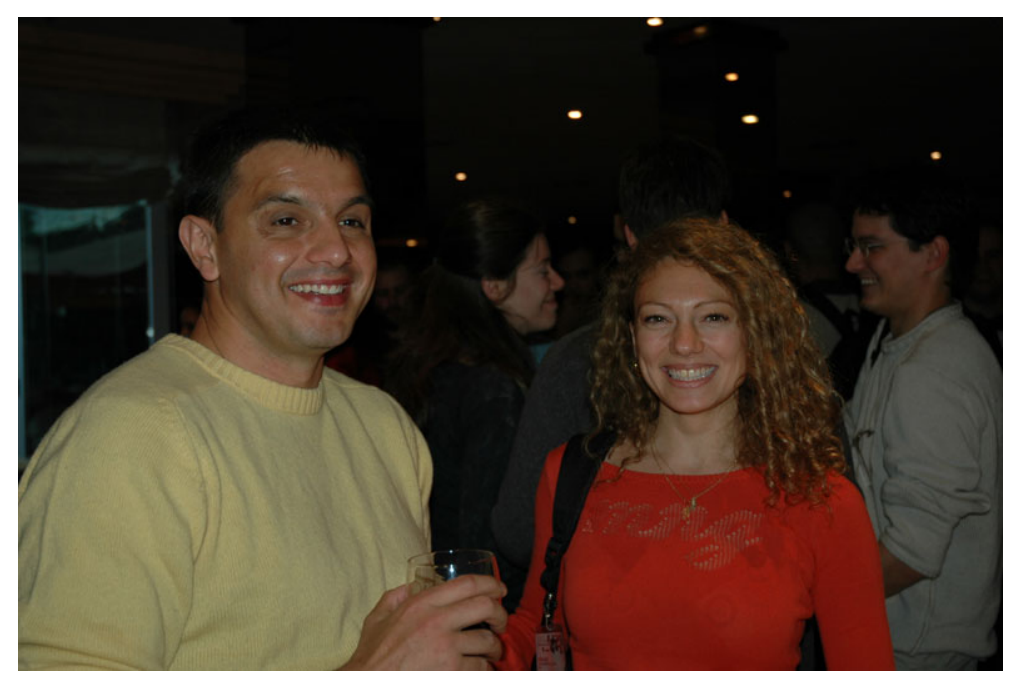

Ricardo Carrera with the speaker.

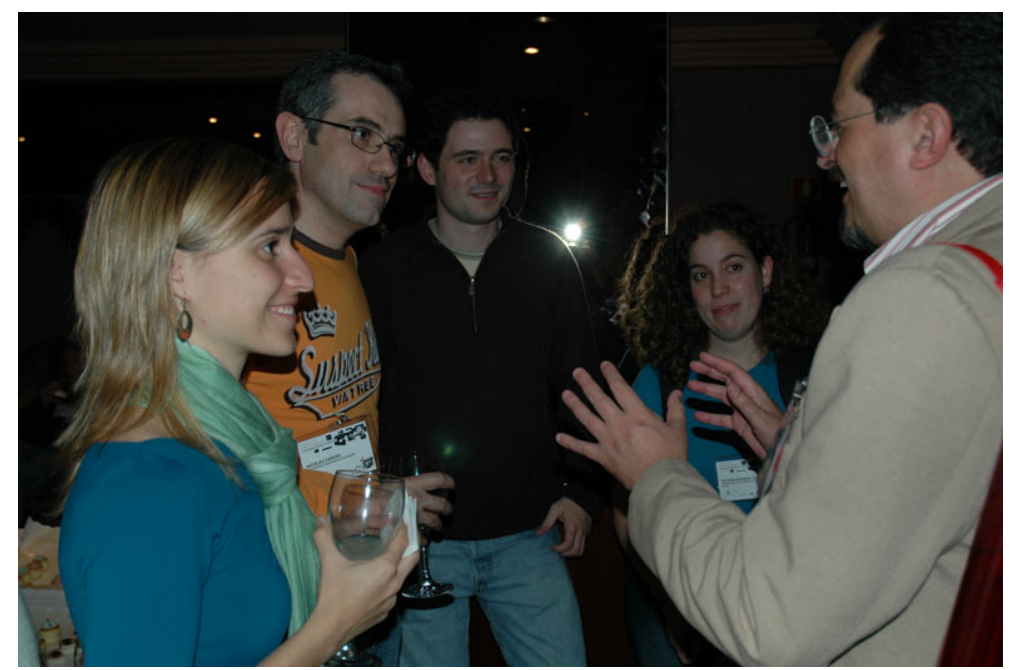

Elisa Toloba, Nicolas Cardiel, Juan Carlos Muñoz Mateos, Esther Marmol and Jesús Gonzalez. 